\title{
Numerical modeling of erosion using an improvement of the extended finite element method
}

\author{
Régis Cottereau* — Pedro Díez ${ }^{* *}$ \\ * Laboratoire MSSMat UMR 8579, École Centrale Paris, CNRS \\ Grande voie des vignes, F-92295 Châtenay-Malabry Cedex, France \\ regis.cottereau@ecp.fr \\ ** Laboratori de Càlcul Numèric, Dept. de Matemàtiques Aplicades III \\ Universitat Politècnica de Catalunya, Barcelona, Spain \\ pedro.diez@upc.edu
}

\begin{abstract}
We present in this paper a numerical model of the erosion of a soil that accounts for both the flow in the open fluid and the flow of fluid through the porous soil. The interface between the open fluid and the soil is represented using a level-set function, and the erosion is controlled by the shear stress vector. The evaluation of the approximate value of this gradient is particularly focused on, and an improved method, called XFE+ method, is presented. Numerical results in $2 D$ and $3 D$ illustrate the accuracy and the potentiality of this method.

RÉSUMÉ. Cet article décrit un modèle numérique d'érosion du sol, qui prend en compte à la fois le fluide dans le sol, considéré comme un milieu poreux, et le fluide extérieur. L'interface entre les deux milieux est représentée à l'aide d'une fonction level-set, et l'érosion est contrôlée par le gradient de la vitesse du fluide. Nous nous concentrons particulièrement sur l'évaluation numérique de ce gradient, et introduisons une méthode, appelée méthode XFE+, pour l'améliorer. Des résultats numériques en $2 D$ et $3 D$ montrent la précision et les potentialités de cette méthode.

KEYWORDS: Erosion, Level-set, XFEM, Darcy's law, Stokes equations, Homogenization MOTS-CLÉS : Érosion, Level-set, XFEM, Équation de Darcy, Équation de Stokes, Homogénéisation
\end{abstract}




\section{Introduction}

The requirement to capture the position and evolution of an interface is central to many areas of engineering and science, including metal forging, oceanography, imaging, flame modeling, melting of materials, and more generally, the modeling of heterogeneous or multi-phases materials. Along the years, several methods have been devised for such problems (Crank, 1984). Among the most used ones are front tracking methods, in which the mesh is refined or deformed to follow the displacement of the interface (Unverdi et al., 1992), and the marker-in-cell method (Gorczyk et al., 2006; Jan, 2007), in which a large set of markers follows the material in a lagrangian way, hence describing the position of each phase. However, both methods induce high computational costs. Indeed, to keep the numerical accuracy to an appropriate level, it is necessary, in the former class of methods, to re-mesh the domain when the deformations increase. Likewise, the number of markers necessary to follow appropriately an interface is shown to be prohibitive for computational implementation (van Keken et al., 1997).
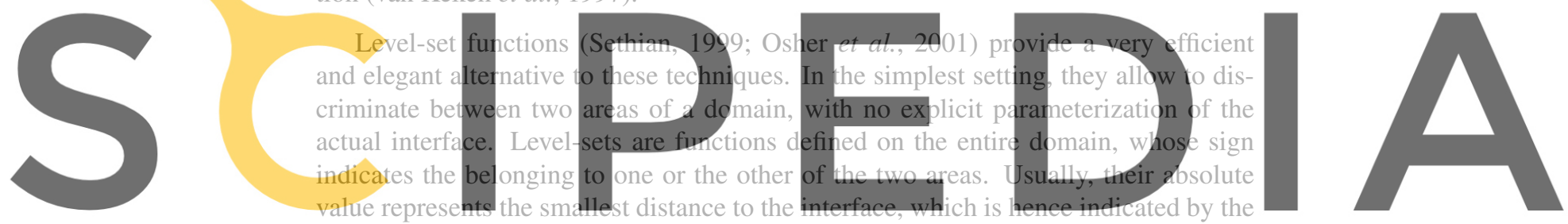

cancellation of the level-set. Conceptually, they are constructed in a space of higher

Since the first use of level-set functions in the description of dynamical two-phase fluid systems (Osher et al., 1988; Sussman et al., 1994), their power has been acknowledged for the parameterization of complex evolving phases. In particular, their ability to deal with changes in topology without any remeshing has been recognized (Mulder et al., 1992; Karlsen et al., 2000). They have been used in several fields of geophysics and geomechanics, including modeling of two-phase flows and permeability estimation in reservoir simulations (Karlsen et al., 2000; Nielsen et al., 2008), tectonic plates subduction (Zlotnik et al., 2007; Zlotnik et al., 2008), seismic waves travel time computation (Sethian et al., 1999), and, generally, for inverse problems and optimal design (Ito et al., 2001; Burger, 2001; Burger et al., 2005).

Following previous papers (Cottereau et al., 2010; Golay et al., 2010), we propose in this paper to use a level-set function to describe a process of erosion. We propose a framework in which both the fluid flow through the soil and the open fluid flow are modeled by the same Brinkman equation, but with physical parameters varying by orders of magnitude. This generalizes and formalizes what was introduced in (Cottereau et al., 2010) for Darcy's law. It can also be seen as the physical counterpart from the more numerical approach in (Golay et al., 2010), based on a penalization method taken from (Angot et al., 1999). The second novel aspect of this paper lies in the numerical 
approach that is used to solve the coupled Brinkman equations problem. We introduce a new method, denoted XFE+ method, that is based on the more classical eXtended Finite Element (XFE) method (Chessa et al., 2003; Moës et al., 2003; Legrain et al., 2008; Zlotnik et al., 2009), but ensures a better evaluation of the fluxes at the interface. As the latter constitutes a classical ingredient of erosion models, this accuracy is of the upmost importance here.

In the next section (section 2), we introduce the erosion model, for which we follow classical lines. We also introduce the three models that are available for the description of fluid flows: Darcy's law, Brinkman equations and Stokes equations. Further, we highlight their relations through homogenization. The main objective of this section is to show that it is reasonable to use the same Brinkman equation on both sides of the eroding interface, but with parameters varying by order of magnitude for the open flow and the porous medium. In section 3, the numerical ingredients of the XFE+ method are introduced, including the level-set functions, Hamilton-Jacobi equation and the different between XFE and XFE+ methods. Finally, two numerical

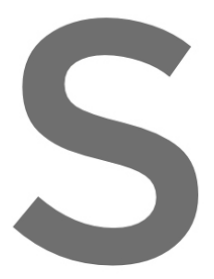
examples are
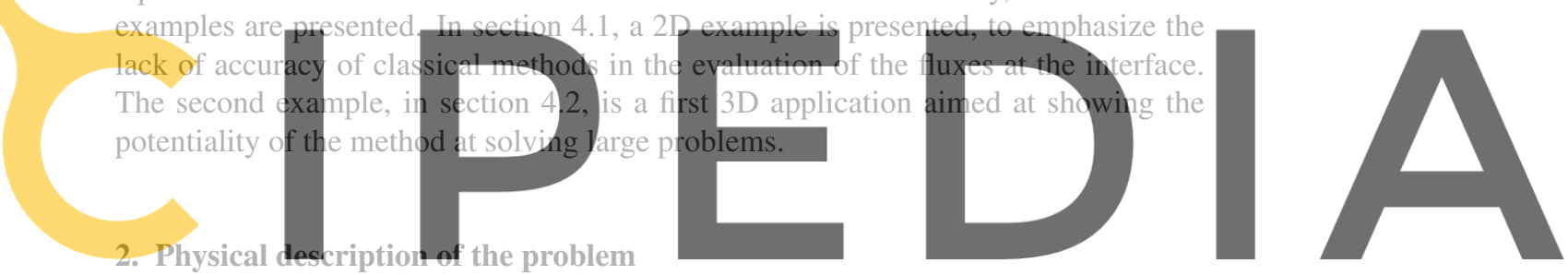

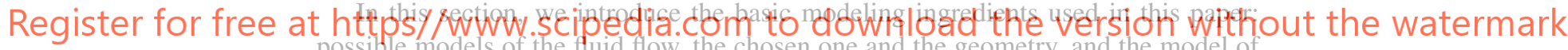 possible models of the fluid flow, the chosen one and the geometry, and the model of}

erosion.

\subsection{Modeling of the fluid flow}

We will consider in this paper three different models for the fluid flow: Darcy and Brinkman's laws, and the Stokes equations. The first one is used in general for the flow of water in a porous medium, the latter is used more often for open fluid flows, and Brinkman's law is an intermediate between the two others. We describe each of these models below, and discuss their relation, with a particular view at homogenization. In figure 1 are sketched both the general setting of a two-phases problem of erosion, where an open flow is coupled to a porous medium, and the particular periodic homogenization setting that is used to derive theoretically Darcy's law and Brinkman equations. 

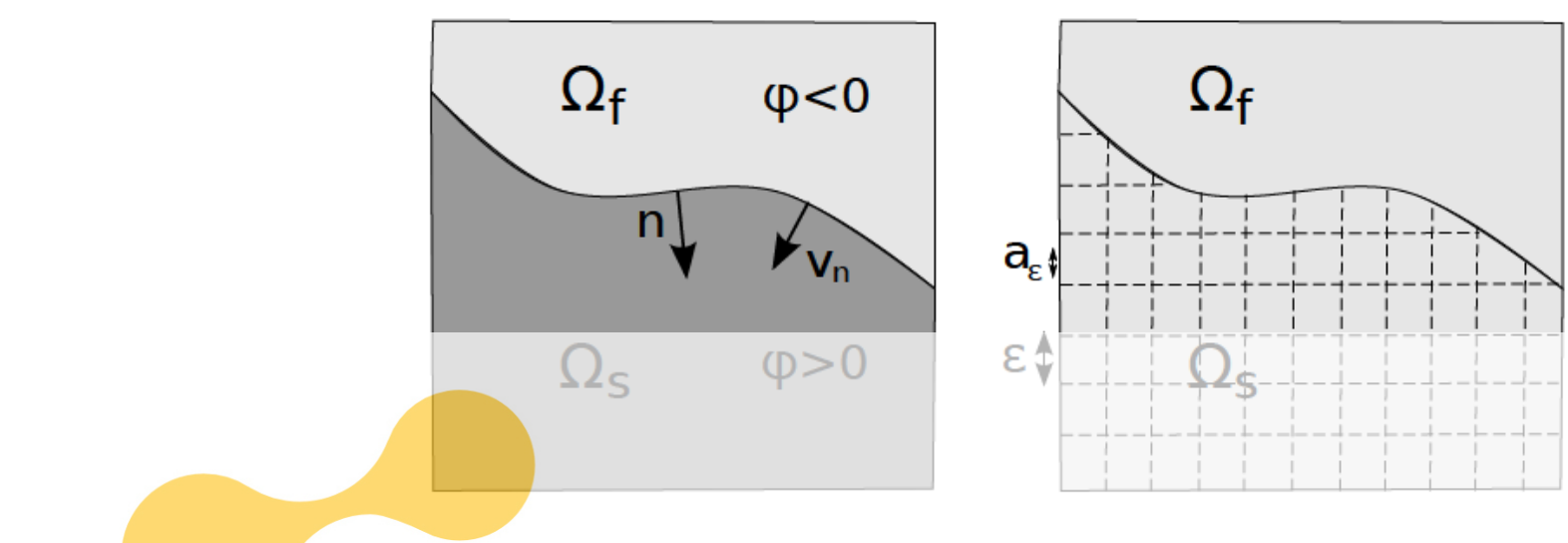

Figure 1. General setting of a two-phases problem (left figure), and detail of the periodic homogenization setting used to derive Darcy and Brinkman equations (right figure)
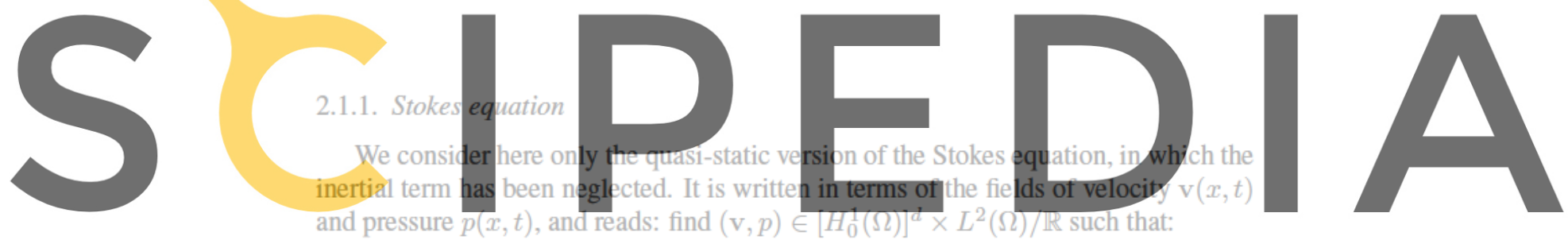

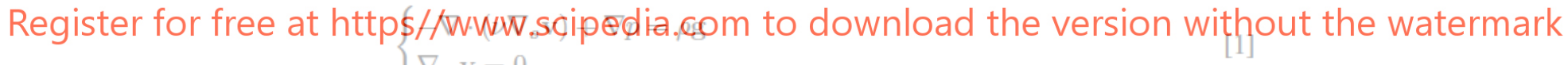

with boundary conditions that will be discussed further. In these equations, $\nu$ is the viscosity of the fluid, $\rho$ its density, $\mathrm{g}$ is the gravitational acceleration vector, and $\nabla_{s}=$ $\left(\nabla+\nabla^{T}\right) / 2$ represents the symmetric part of a gradient. Note that this equation is quasi-static because the inertial terms have been neglected, so that there is no explicit time derivative in the equation. However, because we will consider erosion later on, the domain will be modified with time, and hence the velocity and pressure fields will evolve with time. Incidentally, we will not consider initial conditions, but rather an initial geometry of the domain $\Omega$.

Stokes equations are used to model the flow of a fluid in which advective forces are small compared with viscous forces (low Reynolds number). It is the case for example in flows with slow velocities, large viscosities, or very small length scales.

\subsubsection{Brinkman flow}

When considering the flow of a fluid in a porous medium, Stokes equations can still be used. However, the definition of the precise geometry of all the interfaces between the fluid and the matrix of the medium is then required. When this information is available, which is rarely the case, the cost of the solution of the Stokes equation then 
becomes very large because the details of the geometry have to be accounted for very precisely (Narsilio et al., 2009).

It is therefore often interesting to take into account that influence through the use of a homogenized medium. The matrix of the porous medium is therefore not modeled, and the equations are modified with an additional term that accounts for equivalent drag forces induced on the fluid by the interaction with the matrix. Several authors have derived different methods to estimate this equivalent drag force (Tartar, 1980; Allaire, 1991a; Allaire, 1991b; Jäger et al., 1996). In all of these approaches, the medium is modeled as periodic, with characteristic lengths for the periodicity cell and the matrix inclusions going both to zero at different rates. The homogenization of a random array of impenetrable solid inclusions is treated in (Rubinstein, 1986).

Among others, (Allaire, 1991a) shows that (in 3D, and for homogeneous Dirichlet boundary conditions at the matrix-fluid interfaces), if the size $\epsilon$ of the periodicity cell and the size $a_{\epsilon}$ of the matrix inclusion both tend to zero, such that the ratio $\sigma_{\epsilon}^{2}=$ $\epsilon^{3} / a_{\epsilon}$ tends to a constant value $0<\sigma^{2}<+\infty$, then the solution of the Stokes

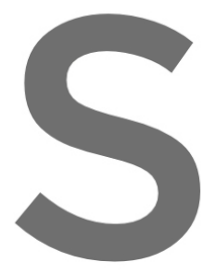

problem converges to the solution of the following Brink $\left[H_{0}^{1}(\Omega)\right]^{d}$
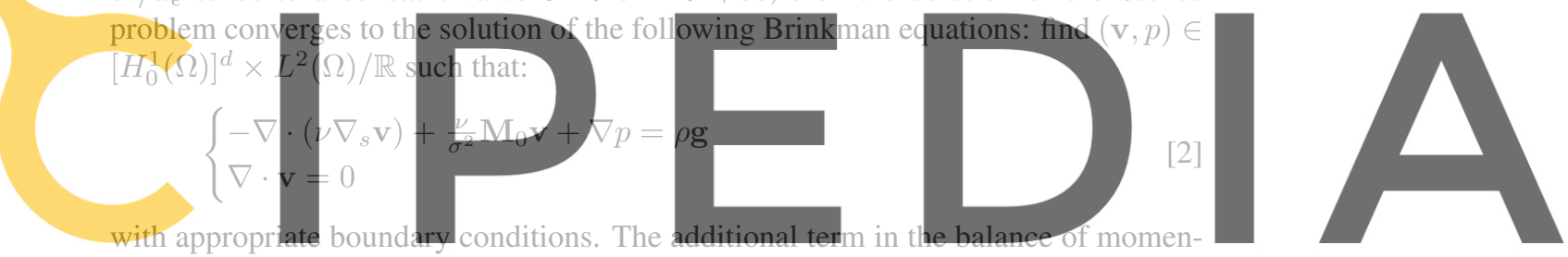

tum represents the homogenized drag force from the matrix onto the fluid. The tensor

boundary conditions at infinity. Note that these equations are homogenized, so both

the velocity and pressures fields, normally defined only in the fluid, are prolongated within the matrix (by zero for the velocity and by an average value for the pressure, see (Allaire, 1991a)).

It is interesting to note that when $\sigma=+\infty$ (meaning that, compared to the Brinkman case, the size of the matrix inclusions are small relatively to the size of the periodicity cells), the Stokes equations are retrieved (Allaire, 1991b). This gives a quantitative definition to the idea that matrix inclusions that are too small cannot influence the fluid flow.

\subsubsection{Darcy's law}

On the other hand, if the rate of convergence of the size of the matrix $a_{\epsilon}$ is larger asymptotically $(\sigma=0)$, the linear term in velocity becomes predominant, and Darcy's law is encountered. More precisely, the rescaled solution $\left(\mathbf{v}_{\epsilon} / \sigma_{\epsilon}^{2}, p_{\epsilon}\right)$ of Stokes equation on the periodic problems with cell size $\epsilon$ can be shown to converge to the solution of the Darcy problem: find $(\mathbf{v}, p) \in\left[H^{1}(\Omega)\right]^{d} \times L^{2}(\Omega) / \mathbb{R}$ such that:

$$
\left\{\begin{array}{l}
\nu \mathbf{M}_{0} \mathbf{v}+\nabla p=\rho \mathbf{g} \\
\nabla \cdot \mathbf{v}=0
\end{array}\right.
$$


with appropriate boundary conditions. The tensor $\mathbf{M}_{0}$ is the same as in the previous section. The scaling of $\mathbf{v}_{\epsilon}$ by $\sigma_{\epsilon}^{2}$ should be understood in the sense that, for a small enough $\epsilon$, the Stokes problem for $\mathbf{v}_{\epsilon}$ really gives the same solution as Darcy's law with a coefficient $\nu \mathbf{M}_{0} / \sigma_{\epsilon}^{2}$.

Darcy's law is the relation of choice for the modeling of filtration of an incompressible fluid through porous media. As for the Stokes case, all inertial terms will be neglected here, although doing otherwise is possible (Bear et al., 1999). Note that this law is usually introduced in terms of the specific discharge $\mathrm{q}=\Phi \mathrm{v}$ and the piezometric head $h=z+p / \rho g$, where $\Phi$ is the volumetric porosity, defined as the ratio between the volume of void space and the total volume of the porous medium, $z$ is the altitude of the considered point, and $g$ is the (scalar) acceleration of gravity (Darcy, 1856): find $(\mathbf{q}, h) \in\left[H^{1}(\Omega)\right]^{d} \times L^{2}(\Omega) / \mathbb{R}$ such that:

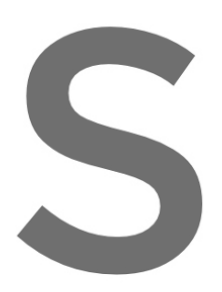

$$
\left\{\begin{array}{l}
\mathrm{q}=-K \nabla h \\
\nabla \cdot \mathrm{q}=0
\end{array}\right.
$$

[4]

where $K$ is the permeability (in unts of velocity). Simple algebra shows that this
formalism is the same as previously, with $\nu \mathbf{M}_{0} / \sigma_{\epsilon}^{2}=(\rho g \Phi / K) \mathbf{I}$, wher $\mathbf{I}$ is the
identity tensor.
Roughly, the permeability describes how easily a fluid flows through a medium,
and its value depends on both the fluid and the medium. Considering the particular
case when $\mathbf{M}_{0}=M_{0} \mathbf{I}$, and using the definition of $\Phi=1-\alpha a_{\epsilon}^{3} / \epsilon^{3}=\left(\sigma_{\epsilon}^{2}-\alpha a_{\epsilon}^{2}\right) / \sigma_{\epsilon}^{2}$
in 3D, with $\alpha$ some proportionality constant depending on the shape of the inclusion

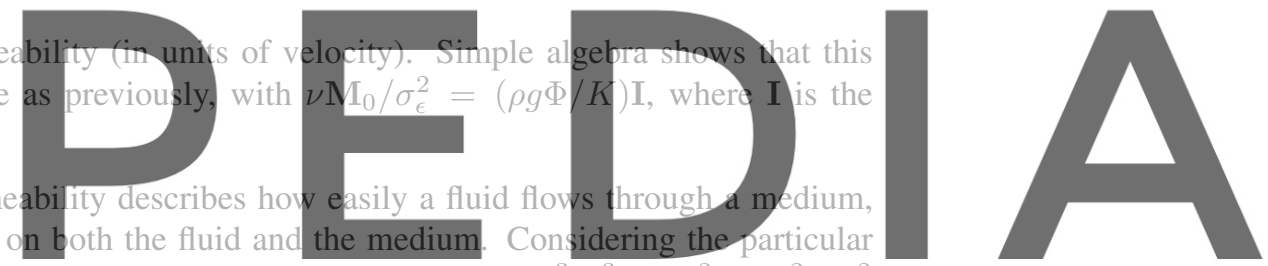

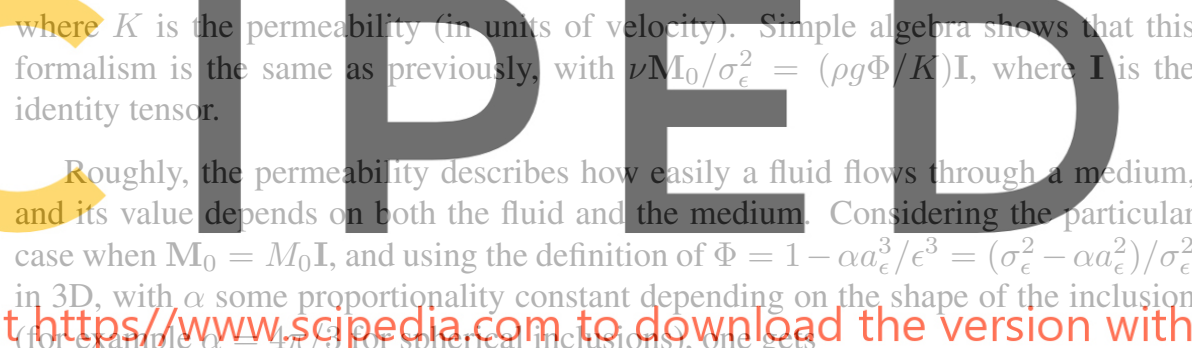

$$
K=\frac{1}{M_{0}} \frac{\rho g}{\nu} \Phi \sigma_{\epsilon}^{2}=\frac{1}{M_{0}} \frac{\rho g}{\nu}\left(\sigma_{\epsilon}^{2}-\alpha a_{\epsilon}^{2}\right) .
$$

In the particular case when $a_{\epsilon}=O(\epsilon)$, which indeed yields the Darcy limit $\sigma=0$, we have $\sigma_{\epsilon}=O\left(\epsilon^{2}\right)$, and we retrieve the analysis of (Hubbert, 1957), obtained from experimental evidence and dimensionality considerations:

$$
K=N \frac{\rho g}{\nu} \epsilon^{2},
$$

where $N$ is a dimensionless factor of proportionality only related to the shape of the passages in the porous medium through which the flow occurs.

\subsubsection{Coupling methods for erosion problems and proposed approach}

The problem that we consider here is that of erosion in a domain $\Omega \in \mathbb{R}^{3}$. That domain is subdivided in a soil domain $\Omega_{s}$, in which there are both soil particles and fluid, and a fluid domain $\Omega_{f}$, where there is only fluid. We assume that $\Omega_{s} \cup \Omega_{f}=\Omega$ and $\Omega_{s} \cap \Omega_{f}=\emptyset$. The fluid domain may be either a cavity or an open area, as in the case of floods for example. Only the fully saturated case is considered for the soil domain. The interface between $\Omega_{s}$ and $\Omega_{f}$, denoted $\Gamma$, is assumed to be a 
discontinuity in the properties, and to be a purely geometrical interface in the sense that it is not attached to any material particle. Indeed, as erosion takes place, this interface evolves. We assume that the quantity of eroded material in the carrier fluid is too small to modify its properties, so that the erosion is only observed through the evolution of the interface.

In these problems of erosion, the open flow is naturally modeled using Stokes equations, while the flow through the porous medium is more often considered through Darcy's law. It is then necessary to design a method for the coupling of these two mathematically heterogeneous equations. This has been investigated by many researchers (Jäger et al., 1996; Discacciati et al., 2009)), with difficulties of a rather mathematical or numerical order. Hypotheses need to be introduced in order for the coupled problem to be solvable. In some cases, these hypotheses simplify the problem greatly and provide (Robin-like) boundary conditions on the Stokes flow (Jäger et al., 1996; Bonelli et al., 2008), rather than a fully coupled problem. However, the physical justification of these hypotheses is sometimes arguable.
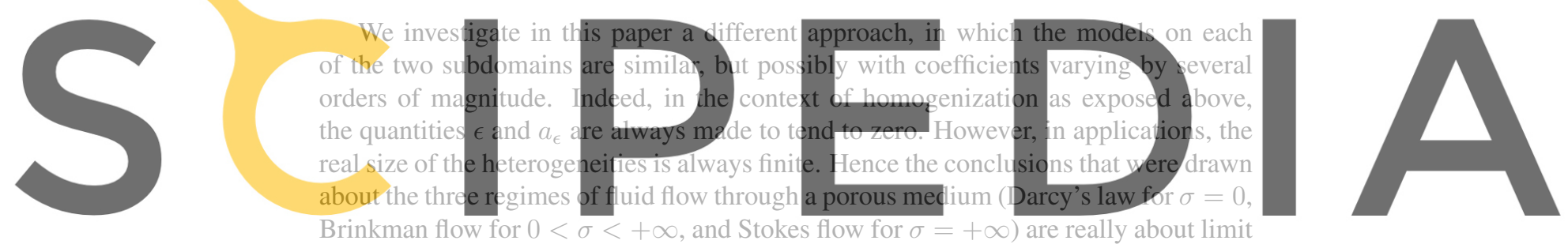

problems. We choose here to use Brinkman equations on both sides of the interface,

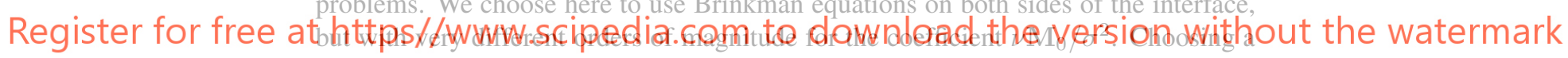

small $\sigma$ on one side would correspond to Darcy's law, while a large $\sigma$ on the other side would lead to Stokes flow. Note that in such an approach, the heterogeneities are never modeled, and the quantities that are derived are really similar to those of Darcy's law (in particular, the pressure and velocity fields are extended within the matrix of the porous medium). Note also that a very mathematical reading of this approach is possible (Angot et al., 1999; Golay et al., 2010), where the coupling of a Stokes flow with a Darcy's law (with a Neumann boundary condition on the pressure) is an $L^{2}$ penalization of that Stokes flow, and the coupling with a Brinkman equation is an $H^{1}$ penalization.

A similar approach for an erosion problem was already considered in (Cottereau et al., 2010), but with Darcy's law rather than Brinkman equations on both sides of the interface. The permeability for the open flow was taken 1000 times larger than the permeability through the soil. In (Vennat et al., 2010), the authors considered Stokes equations on both sides of an interface between an open flow and the flow through a porous medium, and selected the viscosity in the porous medium to be much larger than that in the open area. Although the approach that they consider is similar in spirit to the one that we advocate here, the fact that the viscosities should be different does not appear in the homogenization process that we have described. 


\subsection{Modeling of erosion}

Several models for surface erosion of cohesive soils under axial and radial flow conditions have been proposed in the literature (Vardoulakis et al., 1996; Vardoulakis et al., 2001; Foster et al., 2001; Wan et al., 2004a; Wang et al., 2004; Papamichos et al., 2005). We describe here two models of flow erosion, designed respectively for flows parallel and normal to the surface that is being eroded. The former type of model appears much more often, due to its importance in the creation of gullies and rills, which is an important issue for agriculture in particular (see (Knapen et al., 2007) for a general review). Models dealing with the erosion of a surface under a normal flow are more scarce, and related to problems of the oil industry (Vardoulakis et al., 1996; Vardoulakis et al., 2001). Note that in all the models we discuss here, sedimentation and deposition processes are neglected, and the flows are assumed to be dilute.

Starting with models for perpendicular flows, the most widely used describes the
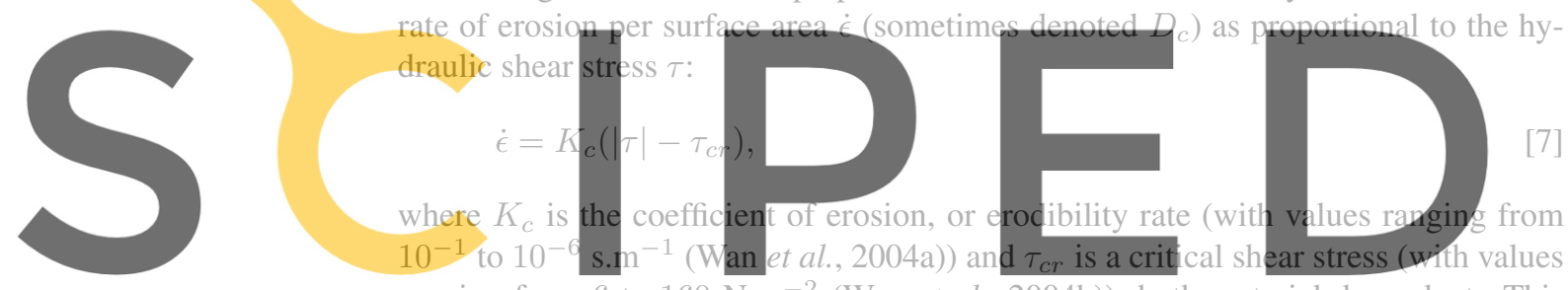

ranging from 6 to $160 \mathrm{~N} \cdot \mathrm{m}^{-2}$ (Wan et al., 2004b)), both material-dependent. This

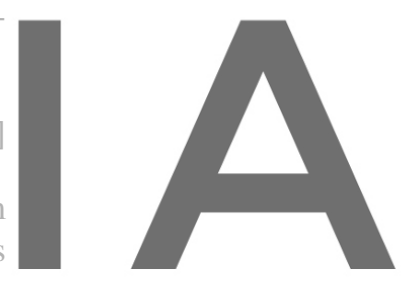

Register for free at https//WwW.scipedia.com to download the version without the watermark $v_{n}(\mathbf{x}, t)=\frac{\dot{\epsilon}}{\rho_{s}}=\frac{K_{c}}{\rho_{s}}\left(|\tau|-\tau_{c r}\right)$.

Other models replace the shear stress by the stream power as the controlling parameter but experimental evidence seems to indicate that the shear force model is appropriate, both for laboratory and in situ cases (Giménez et al., 2002). More refined models try to include the influence of the sediments detached from the soil (see for example (Woodward, 1999; Bonelli et al., 2006; Brivois et al., 2007)), or consider probabilistic models for the erosion factors (Sidorchuk, 2005), however it remains unclear whether the alternatives they provide are truly required with respect to the simpler model of equation [7]. Following (Wan et al., 2004a; Knapen et al., 2007), an approximation can be used to derive the hydraulic shear stress as a function of the gradient of the hydraulic head,

$$
\tau=\rho_{w} g \ell_{0} \nabla h(\mathbf{x}, t)
$$

where $\ell_{0}=1 \mathrm{~m}$ in $2 \mathrm{D}$ problems. This finally leads to a normal velocity for the cavity-soil interface:

$$
v_{n}(\mathbf{x}, t)=\frac{K_{c}}{\rho_{s}}\left(\rho_{w} g \ell_{0}|\nabla h(\mathbf{x}, t)|-\tau_{c r}\right)
$$


where $\rho_{s}$ is the density of the soil material.

In the case of an erosion taking place along the axis of the flow, it is not reasonable to use the shear stress as a controlling parameter for erosion. Following (Vardoulakis et al., 1996; Wang et al., 2004), we get a linear relation between the rate of erosion per surface area and the norm of the gradient of the hydraulic head $\nabla h$, or the fluid velocity $\mathbf{v}$, in the form

$$
v_{n}(\mathrm{x}, t)=\frac{\dot{\epsilon}}{\rho_{s}}=\lambda|\nabla h|=\lambda^{\prime}|\mathrm{v}|,
$$

where $\lambda$ or $\lambda^{\prime}$ are proportionality coefficients, that would have to be determined experimentally. If we neglect $\tau_{c r}$ in equation [10], then the two equations for the normal velocity have the same form (although derived for different origins), but with different proportionality coefficients a priori. As the real flow going through an interface is probably in between that of a normal incidence and grazing incidence, we will consider in all cases a model for the normal velocity as in Eq. [11], with the additional

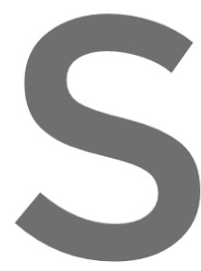

hypothesis th

refined model would require the identification of the coefficient as a function of the angle of incic

3. Numerical description: level-sets and the XFE+ method
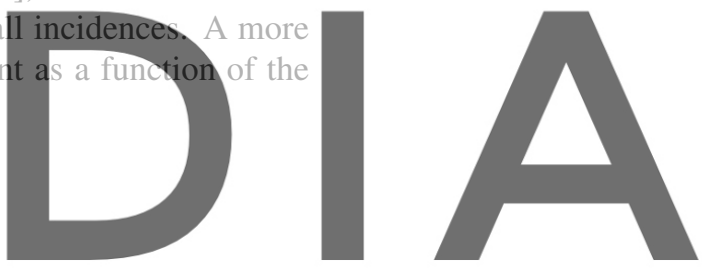

In this section, we present the parameterization of the interface $\Gamma$ between the soil

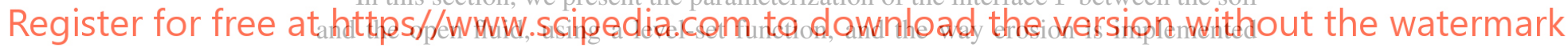

in that context, using the Hamilton-Jacobi equation. This approach has already been used for the modeling of erosion (Cottereau et al., 2010; Golay et al., 2010). We then describe a new numerical method, similar to the XFE method, and called XFE+ method, that improves the accuracy of the approximate solution, both in terms of velocity, and gradient of velocity close to the interface.

\subsection{The level-set description}

As stated in section 2.1.4, the two subdomains $\Omega_{s}$ and $\Omega_{f}$ are separated by an interface $\Gamma$. This interface evolves in time with a normal velocity $\mathbf{v}_{n}(\mathbf{x}, t)$. Note that the definition of this velocity field implies the choice of an "interior" and "exterior".

The main idea of the level-set approach is to consider a function, defined on the entire domain of interest, with values that depend on the position in one phase or the other. The most common approach consists in defining the level-set function $\Phi$ 
as the distance function to the interface, with the sign indicating the subdomain. In mathematical terms, it is defined by

$$
\begin{cases}|\Phi(\mathbf{x})|=\ell(\mathbf{x}) & \\ \Phi(\mathbf{x})>0 & \mathbf{x} \in \Omega_{s} \\ \Phi(\mathbf{x})<0 & \mathbf{x} \in \Omega_{f} \\ \Phi(\mathbf{x})=0 & \mathbf{x} \in \Gamma\end{cases}
$$

where $\ell(\mathrm{x})$ indicates the euclidian distance from point $x$ to the closest point of curve $\Phi=0$. Hence, the level-set function is defined in a space with a higher dimension than the interface it attempts to parameterize. However, the additional dimension allows for an increased smoothness of the function, which can hence be more easily manipulated. Further, the level-set description of the interface fits properly in a FE context because the function $\Phi$ may be interpolated using the same mesh. This allows describing arbitrary interfaces, not restricted to contain the mesh nodes, with an accuracy and regularity up to the resolution of the FE discretization.
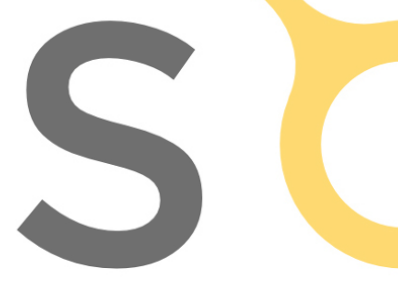

\subsection{The Hamilion-Jacobi equation}

deseription. The evolution of the level-set function $\Phi$ i
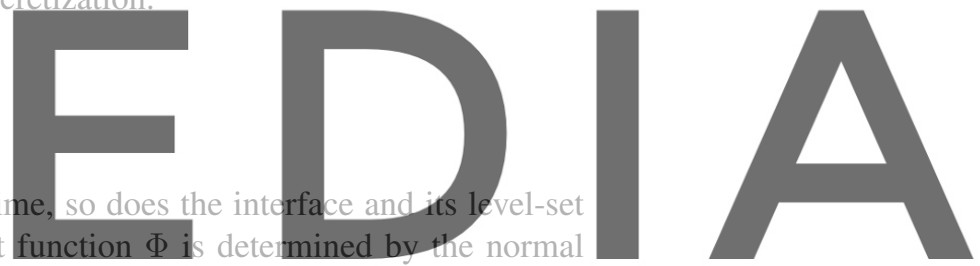

velocity of the interface at every point, $v_{n}(\mathrm{x}, t)$. This front velocity is considered to

Register for free at hetsitive if the interface advances towards the positive yalues of $\Phi$, that is in.the

the Hamilton-Jacobi equation, and its derivation, given the front velocity $v_{n}(\mathrm{x}, t)$, is sketched below.

Let us consider a point $\mathbf{x}(t)$ following the interface in its movement. Thus, at every time $t$,

$$
\Phi(\mathbf{x}(t), t)=0 .
$$

The time derivative of this equation yields

$$
\frac{\partial \Phi}{\partial t}+\nabla \Phi \cdot \frac{\partial \mathbf{x}}{\partial t}=0
$$

Recalling that the front velocity is precisely $v_{n}(\mathbf{x}, t)=\mathbf{n} \cdot \partial \mathbf{x} / \partial t$, with $\mathbf{n}=$ $\nabla \Phi /|\nabla \Phi|$, the second term in the left-hand-side of the previous equation is replaced by $v_{n}(\mathbf{x}, t)|\nabla \Phi|$. This results in the Hamilton-Jacobi equation:

$$
\frac{\partial \Phi}{\partial t}+v_{n}(\mathbf{x}, t)|\nabla \Phi|=0 .
$$

Note that, to this point, no hypothesis has been made on the form of the normal velocity field $v_{n}$. This equation is therefore compatible with different physical models 
of the evolution of the interface. Conversely, the only necessary modification for passing from one model to another is to change the form of the normal velocity. This property was stressed upon in (Cottereau et al., 2010).

\subsection{The XFE+ method}

\subsubsection{Introduction}

In erosion problems, the continuity of the fluxes $\nu \nabla_{s} \mathbf{v} \cdot \mathbf{n}$, along with the discontinuity of the parameter $\nu$ implies a discontinuity of the gradient of the velocity. Hence the only possibility for a FE method to capture adequately the continuity of the fluxes through the interface is by creating a mesh that exactly matches the interface. In that case, the gradients of the solution can be discontinuous between sides of the interface and the fluxes possibly continuous.

However, in many cases, this constraint on the meshing can be an important difficulty. In particular, when considering erosion problems for which the interface evolves, this would mean remeshing fully at each time step. Also, when considering complex geometries, for example arising in mechanics of material when using digitalized images of microstructures, it is much simpler to provide a level set definition of an interface, than to create a mesh exactly fitting it.

It is then possible, within a FE framework, to use the information about the position of the interface to enrich the functional basis in which the approximate solution is sought. This approach leads to the XFE method, in which one adds functions that have a discontinuous gradient over the interface. Thanks to these additional degrees of freedom, the XFE method is theoretically able to match the continuity of fluxes over the interface. However, as will be shown in the application of section 4.1, this is not necessarily the case, because these gradient-discontinuous functions do not provide full freedom in fixing the value of the gradients independently on each side of the interface.

We therefore propose a new method, that enforces explicitly, and in the context of a weak formulation, the continuity of fluxes over the interface. We call this method the XFE+ method, because it uses the same functional basis as the XFE method, but is a mixed formulation with an additional equation enforcing the continuity of fluxes. Only the Brinkman equations are considered below, but the extension to Stokes and Darcy flows is obtained by obvious simplification.

It is important to remember that, in erosion problems (see section 2.2), the most important quantity to be evaluated is the gradient of the solution, because it drives the erosion process. It is therefore very important in these applications that it be well evaluated. This remark is what justifies the discussion in this section, and the subsequent numerical developments. 


\subsubsection{FE and XFE methods}

Starting from the strong formulation of the Brinkman equations in equation [2], the corresponding weak formulation is: find $(\mathbf{v}, p) \in\left[H_{0}^{1}(\Omega)\right]^{d} \times L_{0}^{2}(\Omega)$, such that:

$$
\int_{\Omega} \nu \nabla_{s} \mathbf{v}: \nabla_{s} \mathbf{w} d x+\int_{\Omega} \frac{\nu}{\sigma^{2}} \mathbf{M}_{0} \mathbf{v} \cdot \mathbf{w} d x-\int_{\Omega} p \nabla \cdot \mathbf{w} d x=\int_{\Omega} \rho \mathbf{g} \cdot \mathbf{w} d x
$$

and

$$
-\int_{\Omega} q \nabla \cdot \mathbf{u} d x=0
$$

for all $(\mathbf{w}, q) \in\left[H_{0}^{1}(\Omega)\right]^{d} \times L_{0}^{2}(\Omega)$. The functional spaces are defined by $H_{0}^{1}(\Omega)=$ $\left\{v \in H^{1}(\Omega), v_{\mid \partial \Omega}=0\right\}$, in the case of pure Dirichlet boundary conditions, and $L_{0}^{2}(\Omega)=\left\{p \in L^{2}(\Omega), \int_{\Omega} p d x=p_{0}\right\}$. In the classical FE method, one would choose a mesh of the domain $\Omega$, and (for example) linear functions of approximation on each element of the mesh. If the mesh does not follow the interface $\Gamma$, which is desirable in particular when that interface is evolving, then it is impossible to represent the theoretical discontinuity of the gradient of the solution $\mathbf{v}$ arising from the assumed continuity of the fluxes $\nu \nabla_{s} \mathbf{v}$.

The XFE method (Chessa et al., 2003; Moës et al., 2003; Legrain et al., 2008; Zlotnik et al., 2009) proposes to add to the approximation basis functions that have a discontinuous gradient on the interface $\Gamma$. In terms of global norm of the error, the XFE method behaves much better than the classical FE method, as will be illustrated on an example in section 4.1. However, for the evaluation of the local fluxes on the interface, it does not behave necessarily so well. Indeed, the continuity of fluxes is hidden in the continuous form of the weak formulation [16] but is not enforced anywhere once using the discretized weak formulation.

\subsubsection{XFE+ method}

Trying now to enforce explicitely the continuity of the fluxes over $\Gamma$, and using a Lagrange formulation for instance, one gets the modified weak formulation: find $(\mathbf{v}, p, \lambda) \in\left[H_{0}^{1}(\Omega)\right]^{d} \times L_{0}^{2}(\Omega) \times\left[H_{0}^{1}(\Omega)\right]^{d}$ such that:

$$
\begin{aligned}
& \int_{\Omega} \nu \nabla_{s} \mathbf{v}: \nabla_{s} \mathbf{w} d x-\int_{\Gamma} \llbracket \nu \nabla_{s} \lambda \cdot \mathbf{n} \rrbracket \cdot \mathbf{w} d x+\int_{\Omega} \frac{\nu}{\sigma^{2}} \mathbf{M}_{0} \mathbf{v} \cdot \mathbf{w} d x \\
& -\int_{\Omega} p \nabla \cdot \mathbf{w} d x=\int_{\Omega} \rho \mathbf{g} \cdot \mathbf{w} d x, \\
& -\int_{\Omega} q \nabla \cdot \mathbf{u} d x=0,
\end{aligned}
$$

and

$$
-\int_{\Gamma} \llbracket \nu \nabla_{s} \mathbf{v} \cdot \mathbf{n} \rrbracket \cdot \mu d x=0,
$$


for all $(\mathbf{w}, q, \mu) \in\left[H_{0}^{1}(\Omega)\right]^{d} \times L_{0}^{2}(\Omega) \times\left[H_{0}^{1}(\Omega)\right]^{d}$. In these equations, the jumps over $\Gamma$ are represented with a double bracket:

$$
\llbracket \alpha \rrbracket=\alpha_{\mid \Omega_{1}}-\alpha_{\mid \Omega_{2}} .
$$

As the functional space that is used here is constraining the solution more than in the XFE case, it is expected that the solution will be worse in terms of energy norm. However, in terms of local fluxes close to the interface, thanks to the enforcement of the continuity of the fluxes, which the exact solution verifies, it is expected that the XFE+ solution be better than the XFE one. Indeed, these two statements are shown to hold true on the example in section 4.1.

More considerations about this new method are given in (Cordero et al., 2010; Díez et al., 2010). In particular, the stability of the mixed formulation is proved in the mixed setting above. The applications that we present in the next section are based either on Darcy's law for both the open fluid and the porous medium, or on Stokes equations for both. The corresponding weak formulations for these two cases can be deduced straightforwardly from those above, and the results are qualitatively the same in the Brinkman equations case.

\section{Applications}

We now show two examples of application of the model presented in this paper. First, we discuss a purely numerical example in 2D, where we illustrate the lack of accuracy of both the FE and the XFE methods in evaluating the fluxes on the soilfluid interface, for a Darcy flow on both sides of the interface. Then we present a 3D application of an actual erosion problem, but with Stokes equations on both sides of the interface rather than Brinkman equations. No example will be shown here that uses Brinkman equations, because the corresponding software is still under development. These results will be presented elsewhere. This application is very similar to the one presented in (Golay et al., 2010), but the numerical solution is much faster, because our numerical scheme allows to use a very coarse mesh.

\subsection{Inaccuracy issues in the evaluation of the fluxes at the interface (2D)}

This first example aims at illustrating the lack of accuracy of both the FE and XFE methods in evaluating the fluxes at the interface between two subdomains with different material parameters. As it is of a mathematical nature, and for simplicity, all parameters and variables are here dimensionless, although we still refer to them with their physical names.

Let us consider the open disc $\Omega \in \mathbb{R}^{2}$ centered on $(0,0)$, and with radius 1 . It is split into two concentric subdomains $\Omega_{1}$ (disc) and $\Omega_{2}$ (ring) at radius $1 / 2$, with $\overline{\Omega_{1} \cup \Omega_{2}}=\bar{\Omega}$ and $\Omega_{1} \cap \Omega_{2}=\emptyset$. We use cylindrical coordinates $(r, \theta)$, and define 
the global parameter $\nu$ on $\Omega$ by $\nu\left((r, \theta) \in \Omega_{1}\right)=1000$ and $\nu\left((r, \theta) \in \Omega_{2}\right)=1$. We finally consider the following problem: find $v \in \mathcal{L}^{2}(\Omega)$ such that

$$
\begin{cases}\nu \Delta v+4=0 & , \text { in } \Omega \\ v=0 & , \text { at } r=1\end{cases}
$$

with continuity of velocity and normal flux at the interface. The exact solution $v_{e x}$ of that problem is given by

$$
\left\{\begin{array}{l}
v_{e x}\left(r \leq r_{0}\right)=r^{2} / 1000-1+999 / 4000 \\
v_{e x}\left(r \geq r_{0}\right)=r^{2}-1
\end{array}\right.
$$

The value of the flux, equal in both $\Omega_{1}$ and $\Omega_{2}$ is

$$
\nu \nabla v=2 r \mathbf{e}_{r} .
$$

We compute the approximate solution to this problem using three different meshes (see figure 2) and three different methods: the FE method, the classical XFE method, and the XFE+ method.

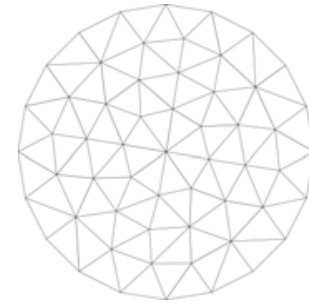

(a) $\mathrm{N}=92$

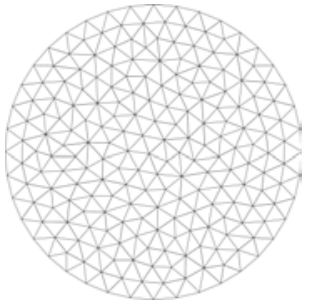

(b) $\mathrm{N}=392$

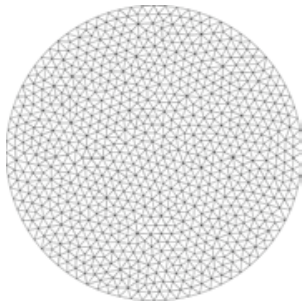

(c) $\mathrm{N}=1568$

Figure 2. Sequence of meshes used for the numerical simulations. $N$ indicates the number of (triangular) elements in each mesh

In figure 3, we present, for each mesh and each method, element maps of the normalized error in norm $H^{1}$, defined by

$$
e_{k}=\frac{\left\|v_{H}-v_{e x}\right\|_{H^{1}, T_{k}}}{\left\|v_{e x}\right\|_{H^{1}, T_{k}}}
$$

with $\|v\|_{H^{1}, T}^{2}=\int_{T} \nabla v \cdot \nabla v d x$ the square of the $H^{1}$ norm of function $v$ over domain $T$. The comparison between the three methods shows that there is an important increase of accuracy when switching from the FE method to the XFE method. Indeed, the addition in the functional basis for the approximate solution of an element with discontinuity of the gradient at the interface apparently to allows to capture the fluxes perfectly in that location. Hence in both the XFE and the XFE+ methods, the major 
error (normalized) arises from the central point. However, the theoretical improvement of the gradients at the interface does not seem to be observable on these maps, except slightly for the first mesh. The evolution of the global error in $H^{1}$ norm

$$
e_{\Omega}=\frac{\left\|v_{H}-v_{e x}\right\|_{H^{1}, \Omega}}{\left\|v_{e x}\right\|_{H^{1}, \Omega}}=\sqrt{\frac{3000}{7001 \pi}}\left\|v_{H}-v_{e x}\right\|_{H^{1}, \Omega},
$$

is also plotted in figure 4 (left figure), and yields the same conclusion that XFE and $\mathrm{XFE}+$ seem equivalent.

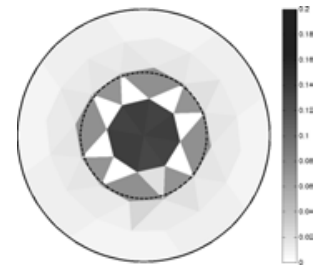

(a) FE, Mesh 1

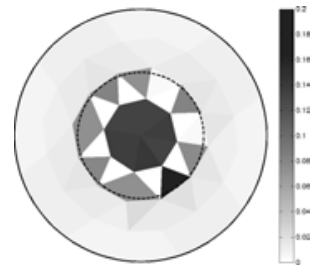

(d) XFE, Mesh 1

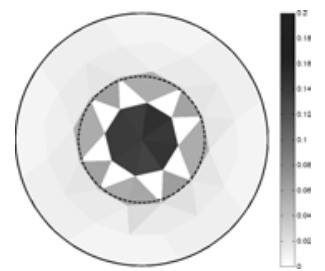

(g) XFE+, Mesh 1

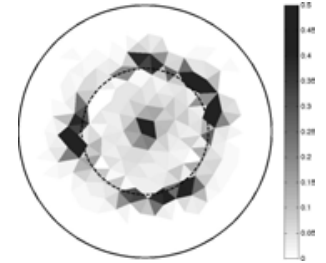

(b) FE, Mesh 2

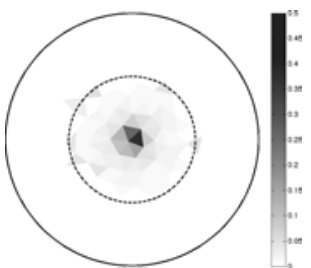

(e) XFE, Mesh 2

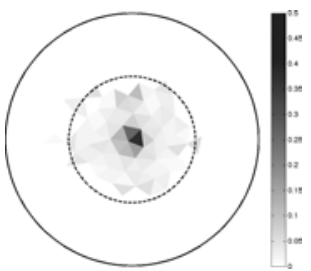

(h) XFE+, Mesh 2

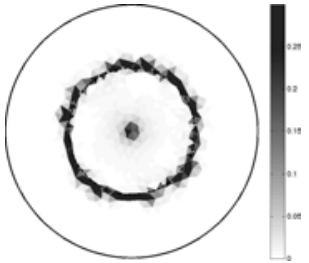

(c) FE, Mesh 3

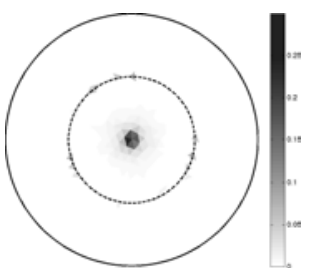

(f) XFE, Mesh 3

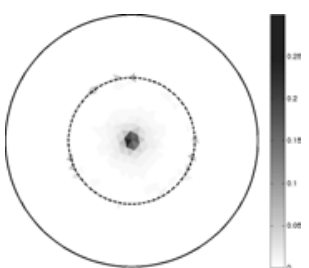

(i) $\mathrm{XFE}+$, Mesh 3

Figure 3. Elemental contributions to the normalized error in norm $H^{1}$, on three different meshes and with the three different numerical methods, FE, XFE, and XFE+. The dashed circle indicates the position of the interval. The color scales are the same for all methods and one given mesh but is different for different meshes

However, on figure 4 , we observe directly the evolution of the normalized error in $H^{1 / 2}$ norm over the interface. This error is defined as

$$
e_{\Gamma}=\frac{\left\|v_{H}-v_{e x}\right\|_{H^{1 / 2}, \Gamma}}{\left\|v_{e x}\right\|_{H^{1 / 2}, \Gamma}}=\frac{\nu}{\sqrt{\pi}}\left\|v_{H}-v_{e x}\right\|_{H^{1 / 2}, \Gamma},
$$

with $\|v\|_{H^{1 / 2}, L}^{2}=\int_{L} \nabla v \cdot \nabla v d x$ the square of the $H^{1 / 2}$ norm of function $v$ over the boundary $L$. As the functions $v_{H}$ and $v_{e x}$ are discontinuous over the interface $\Gamma$, we 
compute two different errors: one (center plot) using the values of the functions on the side of $\Omega_{1}, e_{\Gamma, 1}$, and one (right plot) using the values on the side of $\Omega_{2}, e_{\Gamma, 2}$. It can be observed that, although the evaluation of the fluxes was well performed considering the $\Omega_{2}$ side (on which $\nu$ is the smallest), this is not so on the other side, where the fluxes are very badly evaluated by the XFE method, and better by the XFE+ method. In the XFE method, the gradients of the velocity are more poorly evaluated on the side where the parameter $\nu$ is larger.
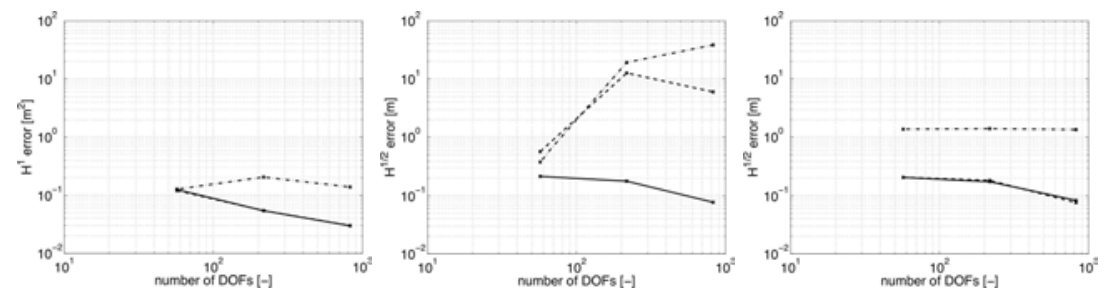

Figure 4. Convergence of the normalized error in $H^{1}$ norm (left) over $\Omega$ and in $H^{1 / 2}$ norm over $\Gamma_{1}$ (center) or $\Gamma_{2}$ (right), in terms of the number of degrees of freedom of the mesh, for the FE (dash-dotted), the XFE (dashed line, confounded with the solid line on the left and right plots), and the XFE+ (solid line) methods

Hence, as expected and described in section 3.3, the FEM behaves poorly both in terms of global energy norm and local fluxes, the XFE method behaves better in terms of energy norm and as poorly in terms of local fluxes, and the XFE+ method behaves better in terms of local fluxes. Also, in terms of local fluxes, the XFE method behaves better than the XFE+ method, but, as can be seen on the example, very slightly so. On the other hand, the improvement in terms of local fluxes is very sensible.

\subsection{Erosion around a $3 D$ bridge pier}

We consider here a 3D example very similar to the one described in (Golay et al., 2010) about the evaluation of erosion around a bridge pier. We therefore consider a 3D box, with water on the upper side, and a water-filled soil in the lower part. Stokes equations are used, both for the open water and the flow through the porous soil. The normal velocity is imposed on two of the sides, while wall conditions are imposed on the rest of the sides. This creates a flow of water through the system, which accelerates around the pier, because of the decreased area. A level-set was used to represent the interface between the soil and the open flow, while the pier was explicitly meshed (hence, the pier is taken as a boundary to the domain). A coarse mesh of 1989 nodes and 8858 tetrahedra was used and the simulation ran in $1 \mathrm{~h} 30$ on a basic dual-core laptop. This is much less than the 2 days reported in (Golay et al., 2010), and this gain is obviously related to the important coarsening of the mesh (200 times less degrees of freedom). Although it is hard to compare the two 
computational methods very precisely because some data is missing in the paper, our method appears very promising for 3D applications.

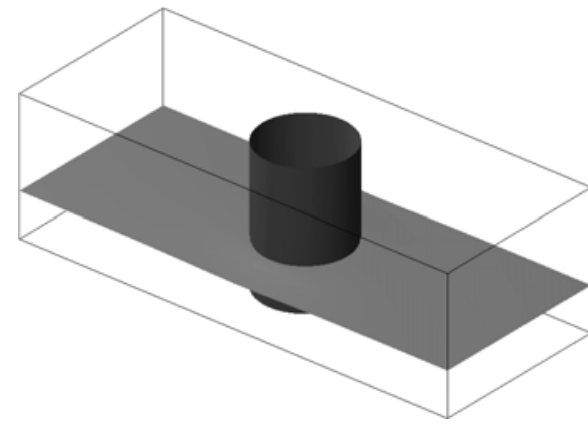

(a) $t_{1}$

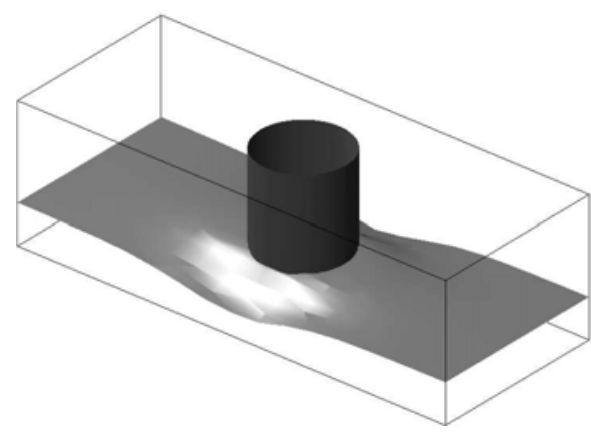

(c) $t_{3}$

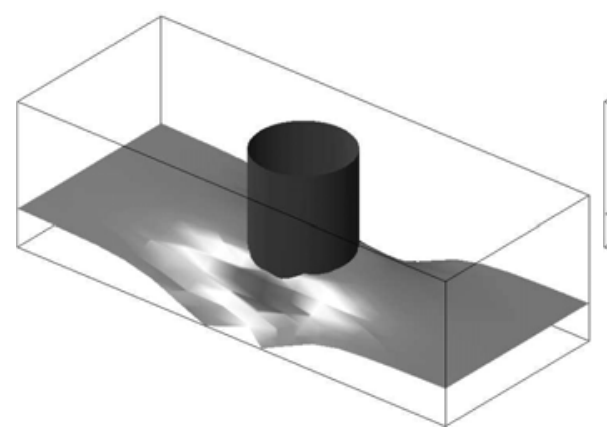

(e) $t_{5}$

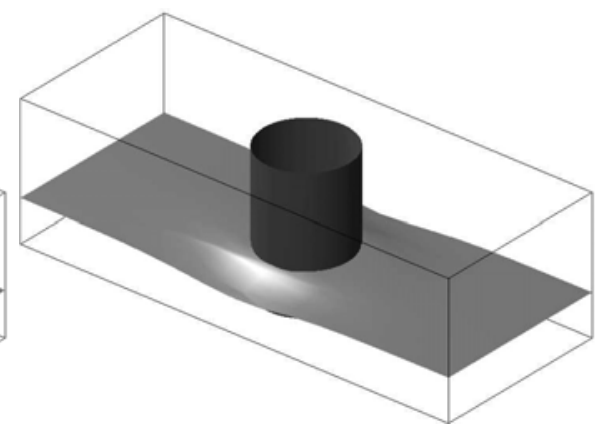

(b) $t_{2}$

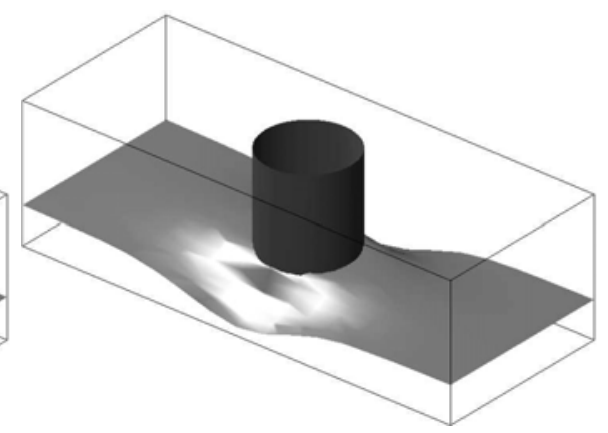

(d) $t_{4}$

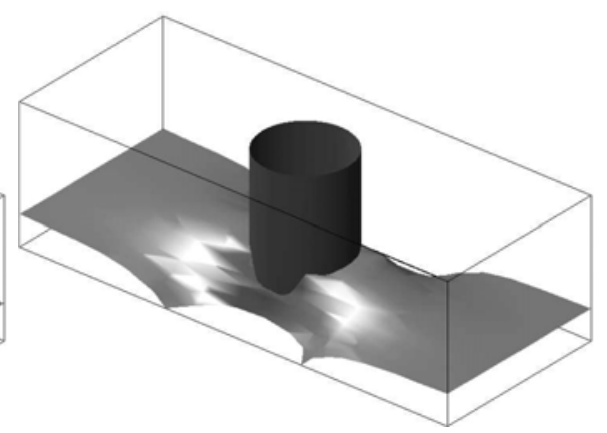

(f) $t_{6}$

Figure 5. Evolution of the fluid-soil interface with time for the pier example. The pier is represented by the dark cylinder, while the greyish surface is the interface 


\section{Conclusions}

We have presented in this paper a numerical model of erosion of a porous medium at the interface with an open flow. It is based on two ingredients: the use of Brinkman equations for both the open flow and the flow through the porous medium, with very different mechanical parameters on the two sides of the interface, and a new XFE+ method to evaluate the shear force at the interface accurately. Although the results, in terms of both efficiency and accuracy are very promising, it is now necessary to finalize the implementation of the Brinkman equations (the examples shown here were with Darcy's law and Stokes equations).

\section{References}

Allaire G., " Homogenization of the Navier-Stokes equations in open sets perforated with tiny holes I. Abstract framework, a volume distribution of holes", Archives for Rational Mechanics and Analysis, vol. 113, n 3, p. 209-259, 1991a.

Allaire G., " Homogenization of the Navier-Stokes equations in open sets perforated with tiny holes II. Non-critical sizes of the holes for a volume distribution and a surface distribution of holes", Archives for Rational Mechanics and Analysis, vol. 113, n 3, p. 261-298, 1991b.

Angot P., Bruneau C.-H., Fabrie P., “ A penalization method to take into account obstacles in incompressible viscous flows", Numerische Mathematik, vol. 81, n 4, p. 497-520, 1999.

Bear J., Bachmat Y., " Introduction to modeling of transport phenomena in porous media", in J. Bear (ed.), Theory and Applications of Transport in Porous Media, vol. 4, Kluwer Academic Publishers, p. 136, 1999.

Bonelli S., Brivois O., " The scaling law in the hole erosion test with a constant pressure drop”, International Journal for Numerical and Analytical Methods in Geomechanics, vol. 32, $\mathrm{n}^{\circ} 13$, p. 1573-1595, 2008.

Bonelli S., Brivois O., Borghi R., Benahmed N., “ On the modelling of piping erosion”, Comptes-Rendus Mécanique, vol. 334, n 8-9, p. 555-559, 2006.

Brivois O., Bonelli S., Borghi R., " Soil erosion in the boundary layer flow along a slope: a theoretical study”, European Journal of Mechanics B/ Fluids, vol. 26, p. 707-719, 2007.

Burger M., “ A level set method for inverse problems”, Inverse Problems, vol. 17, n 5, p. $1327-$ 1355, 2001.

Burger M., Osher S. J., “ A survey of level set methods for inverse problems and optimal design”, European Journal of Applied Mathematics, vol. 16, n 2, p. 263-301, 2005.

Chessa J., Belytschko T., “ An extended finite element method for two-phase fluids: flow simulation and modeling”, Journal of Applied Mechanics, vol. 70, n 1, p. 10-17, 2003.

Cordero F., Díez P., “ XFEM+: una modificación de XFEM para mejorar la precisión de los flujos locales en problemas de difusión con conductividades muy distintas", Revista Internacional de Métodos Numéricos para Cálculo y Diseño en Ingeniería, vol. 26, $\mathrm{n}^{\circ} 2$, p. 121-133, 2010.

Cottereau R., Díez P., Huerta A., “ Modeling, with a unified level-set representation, of the expansion of a hollow in the ground under different physical phenomena”, Computational Mechanics, vol. 46, $\mathrm{n}^{\circ}$ 2, p. 315-327, 2010. 
Crank J., Free and moving boundary problems, Oxford University Press, 1984.

Darcy H., Les Fontaines Publiques de la Ville de Dijon, Dalmon, Paris, 1856.

Díez P., Cottereau R., Cordero F., "XFEM+: a modification of XFEM to improve on the accuracy of local fluxes in diffusion problems with very different conductivities”, 2010. in preparation.

Discacciati M., Quarteroni A., “Navier-Stokes/Darcy coupling: modeling, analysis and numerical approximation”, Revista Matemática Complutense, vol. 22, n² 2, p. 315-426, 2009.

Foster M., Fell R., " Assessing embankment dam filters that do not satisfy design criteria", Journal of Geotechnical and Geoenvironmental Engineering, ASCE, vol. 125, n 7, p. 398407, 2001.

Giménez R., Govers G., "Flow detachment by concentrated flow on smooth and irregular beds", Soil Science Society of America Journal, vol. 66, p. 1475-1483, 2002.

Golay F., Lachouette D., Bonelli S., Seppecher P., “ Interfacial erosion: a three-dimensional numerical model”, Comptes-Rendus Mécanique, vol. 338, nº 6, p. 333-337, 2010.

Gorczyk W., Gerya T. V., Connolly J. A., Yuen D. A., Rudolph M., “ Large-scale rigid-body rotation in the mantle wedge and its implications for seismic tomography", Geochemistry Geophysics Geosystems, vol. 7, n 5, p. Q05018, 2006.

Hubbert M. K., “ Darcy's law and the field equation of the flow of underground fluids”, Hydrological Sciences Journal, vol. 2, n 1, p. 23-59, 1957.

Ito K., Kunisch K., Li Z., “'Level-set function approach to an inverse interface problem”, Inverse Problems, vol. 17, n 5, p. 1225-1242, 2001.

Jäger W., Mikelić A., " On the boundary conditions at the interface between a porous medium and a free fluid”, Ann. Scuola Norm. Sup. Pisa Cl. Sci., vol. 12, p. 403-465, 1996.

Jan Y.-J., " A cell-by-cell thermally driven mushy cell tracking algorithm for phase-change problems”, Computational Mechanics, vol. 40, n² 2, p. 201-216, 2007.

Karlsen K. H., Lie K.-A., Risebro N. H., “ A fast marching method for reservoir simulation”, Computational Geosciences, vol. 4, n², p. 185-206, 2000.

Knapen A., J.Poesen, Govers G., Gyssels G., Nachtergaele J., " Resistance of soils to concentrated flow erosion: a review”, Earth-Science Reviews, vol. 80, n 1-2, p. 75-109, 2007.

Legrain G., Moës N., Huerta A., " Stability of incompressible formulations enriched with XFEM", Computer Methods in Applied Mechanics and Engineering, vol. 197, $\mathrm{n}^{\circ} 21-24$, p. 1835-1849, 2008.

Moës N., Cloirec M., Cartraud P., Remacle J.-F., “ A computational approach to handle complex microstructure geometries”, Computer Methods in Applied Mechanics and Engineering, vol. 192, n 28-30, p. 3163-3177, 2003.

Mulder W., Osher S., Sethian J. A., “ Computing interface motion in compressible gas dynamics”, Journal of Computational Physics, vol. 100, n 2, p. 209-228, 1992.

Narsilio G. A., Buzzi O., Fityus S., Yun T. S., Smith D. W., “Upscaling of Navier-Stokes equations in porous media: theoretical, numerical and experimental approach", Comp. Geotech., vol. 36, p. 1200-1206, 2009.

Nielsen L. K., Li H., Tai X.-C., Aanonsen S. I., Espedal M., “ Reservoir description using a binary level set model”, Computing and Visualization in Science, 2008.

Osher S., Fedkiw R. P., “ Level set methods: an overview and some recent results”, Journal of Computational Physics, vol. 169, n² 2, p. 463-502, 2001. 
Osher S., Sethian J. A., “ Fronts propagating with curvature-dependent speed: Algorithms based on Hamilton-Jacobi formulations", Journal of Computational Physics, vol. 79, $n^{\circ}$ 1, p. 1249, 1988.

Papamichos E., Vardoulakis I., " Sand erosion with a porosity diffusion law", Comp. Geotech., vol. $32, \mathrm{n}^{\circ} 1$, p. 47-58, 2005.

Rubinstein J., " On the macroscopic description of slow viscous flow past a random array of spheres", Journal of Statistical Physics, vol. 44, n 5-6, p. 849-863, 1986.

Sethian J. A., Level set methods and fast marching methods, Cambridge University Press, 1999.

Sethian J. A., Popovici A. M., " 3-D traveltime computation using the fast marching method", Geophysics, vol. 64, n², p. 516-523, 1999.

Sidorchuk A., "Stochastic components in the gully erosion modelling", CATENA, vol. 63, n ${ }^{\circ} 2-$ 3, p. 299-317, 2005.

Sussman M., Smereka P., Osher S., " A level set approach for computing solutions to incompressible two-phase flow", Journal of Computational Physics, vol. 114, n 1, p. 146-159, 1994.

Tartar L., Non-homogeneous media and vibration theory, Lectures Notes in Physics, Springer, chapter Incompressible fluid flow in a porous medium: convergence of the homogenization process, p. 368-377, 1980.

Unverdi S. O., Tryggvason G., “ A front-tracking method for viscous, incompressible, multifluid flows”, Journal of Computational Physics, vol. 100, n 1, p. 25-37, 1992.

van Keken P. E., King S. D., Schmeling H., Christensen U. R., Neumeister D., Doin M.-P., “ A comparison of methods for the modeling of thermochemical convection", Physics of Fluids $A$, vol. 102, n B10, p. 22477-22496, 1997.

Vardoulakis I., Papanastasiou P., Stavropoulou M., "Sand erosion in axial flow conditions", Transport in Porous Media, vol. 45, n 2, p. 267-280, 2001.

Vardoulakis I., Stavropoulou M., Papanastasiou P., " Hydro-mechanical aspects of the sand production problem”, Transport in Porous Media, vol. 22, n 2, p. 225-244, 1996.

Vennat E., Aubry D., Degrange M., “ Collagen fiber network infiltration: permeability and capillary infiltration”, Transport in Porous Media, vol. 84, n 3, p. 717-733, 2010.

Wan C. F., Fell R., “ Investigation of rate of erosion of soils in embankment dams”, Journal of Geotechnical and Geoenvironmental Engineering, ASCE, vol. 130, n 4, p. 373-380, 2004a.

Wan C. F., Fell R., “ Laboratory tests on the rate of piping erosion of soils in embankment dams”, Geotechnical Testing Journal, vol. 27, n³ 3, p. 1-9, $2004 \mathrm{~b}$.

Wang J., Wan R. G., “ Computation of sand fluidization phenomena using stabilized finite elements", Finite Elements Anal. Design, vol. 40, n 12, p. 1681-1699, 2004.

Woodward D. E., “"Method to predict cropland ephemeral gully erosion”, CATENA, vol. 37, n 3-4, p. 393-399, 1999.

Zlotnik S., Díez P., " Hierarchical X-FEM for n-phase flow $(\mathrm{n}>2)$ ”, Computer Methods in Applied Mechanics and Engineering, vol. 198, n³0-32, p. 2329-2338, 2009.

Zlotnik S., Díez P., Fernández M., Vergés J., “ Numerical modelling of tectonic plates subduction using X-FEM", Computer Methods in Applied Mechanics and Engineering, vol. 196, $\mathrm{n}^{\circ}$ 41-44, p. 4283-4293, 2007.

Zlotnik S., Fernández M., Díez P., Vergés J., “ Modelling gravitational instabilities: slab breakoff and Rayleigh-Taylor diapirism”, Pure and Applied Geophysics, vol. 165, p. 1-20, 2008. 